\title{
DESAFÍOS ACTUALES PARA LA ENSEÑANZA DE LAS CIENCIAS ${ }^{1}$
}

\section{Current Challenges for the Teaching of Science}

\author{
Marco Antonio Moreira \\ https://orcid.org/0000-0001-7484-1548 \\ Instituto de Física, Universidade Federal do Rio Grande do Sul - FRGS \\ Porto Alegre, RS, Brasil \\ moreira@if.ufrgs.br
}

Recibido: 27 de mayo de 2021

Aceptado: 30 de junio de 2021

Resumen: La enseñanza de las ciencias es abordada desde una perspectiva crítica y de desafíos en la educación contemporánea. La idea subyacente es que esa enseñanza debería ocuparse más de un aprendizaje significativo, y para la ciudadanía, de las ciencias en vez de una preparación para los test locales, nacionales e internacionales, es decir la enseñanza para los test.

Palabras clave: enseñanza de las ciencias, aprendizaje significativo, enseñanza para los test.

Abstract: The teaching of science is approached from a critical perspective and from contemporary challenges. The underlying ideas are that this teaching should give more attention to a meaningful learning, and to citizenship, of science instead of a teaching for testing at local, national, and international levels.

Keywords: science teaching, meaningful learning, teaching for testing.

\section{Las ciencias en nuestra vida}

La Física, por ejemplo, permea toda la vida de un(a) ciudadano(a). Está en la base de las tecnologías de información, de la ingeniería, de técnicas de diagnóstico y tratamiento usadas en la medicina.

Física tiene modelos y teorías que explican gran parte del mundo físico en que vivimos. Biología, Química, Neurociencia y otras áreas científicas utilizan conceptos, principios, modelos y teorías derivadas de la Física, pero tienen identidad propia.

\section{Aprender ciencias}

Obviamente, aprender ciencias (Física, Química, Biología y afines) es un derecho de la ciudadanía. Una pedagogía libertadora debe sacar el ser humano del sentido común, de interpretaciones ingenuas, de la aceptación ciega de teorías y modelos.

1 Conferencia inaugural de las I Jornadas Académicas de Enseñanza de las Ciencias Naturales, 3 y 4 de diciembre de 2020, Montevideo, Uruguay. 


\section{Las ciencias, ¿cómo son?}

Las ciencias dicen no al sentido común, a las interpretaciones ingenuas, a la aceptación acrítica de teorías y modelos.

Las interpretaciones científicas nunca son definitivas. En ciencias hay una búsqueda permanente de mejores modelos y teorías para explicar el Universo desde perspectivas subatómicas hasta macro cósmicas, así como para explicar fenómenos químicos y biológicos con los cuales convive el ser humano.

\section{Enseñanza de las ciencias}

Por lo tanto, si las ciencias son así y si su aprendizaje es libertador, su enseñanza debe facilitar el aprendizaje significativo de sus contenidos y procedimientos. ¿Eso pasa? ¿Cómo ocurre eso en la realidad de un salón de clases?

\section{Grandes desafíos en la enseñanza de las ciencias}

- En 2013, la revista Science ha dedicado gran parte de un volumen al tema Grandes Desafíos en la Enseñanza de las Ciencias (Grand Challenges in Science Education, Science, 19 de abril de 2013, pp. 290-323). En las primeras páginas de este material, Carl Wieman, Premio Nobel de Física en 2011 y muy activo en la enseñanza de las ciencias, dijo con destaque, refiriéndose a la enseñanza superior:

TRANSFORMACIÓN ES POSIBLE SI LA UNIVERSIDAD REALMENTE QUIERE

\section{La enseñanza de ciencias en la universidad}

La manera como la mayoría de las universidades de investigación enseña ciencias en los cursos de grado es peor que ineficaz. Es no científica. (op.cit., p. 292).

Hay toda una industria dedicada a medir cuán importante es mi investigación, con factores de impacto de mis artículos y por ahí va. Sin embargo, ni siquiera recogen datos sobre cómo estoy enseñando. Eso no recibe atención. (op.cit., p. 293).

Hay muchos profesores a los que les parece totalmente apropiado dedicar más tiempo mejorando su enseñanza, pero no es eso lo que se espera de ellos. (ibid.)

\section{La enseñanza de las ciencias}

De hecho, es así. En carreras como, Ingeniería, por ejemplo, los estudiantes miran la Física como obligación, no como una disciplina fundamental en su formación. Lo que quieren es pasar y verse libres de la Física.

En enseñanza media es peor porque los alumnos acaban "odiando" la Física.

¿Qué pasa? La Física, por ejemplo, es enseñada como si fuera un montón de fórmulas y respuestas correctas. Para cada problema existe una fórmula y para cada pregunta existe una respuesta correcta. 
Eso no es Física, pero es así enseñada entrenando a los estudiantes a resolver problemas usando fórmulas "mágicas" y dar respuestas correctas en los test.

Lo mismo puede pasar en la enseñanza de la Química, con reacciones y fórmulas, y en la de Biología, con taxonomías y ...

Significados, comprensión, modelaje, criticidad, no son requisitos. Aprendizaje mecánico de soluciones de problemas y respuestas correctas sí, en esa enseñanza para los test (el teaching for testing).

Aunque el discurso sea otro, muchas veces muy bonito, ese tipo de enseñanza no lleva a una adquisición de conocimientos para la ciudadanía.

Cuando los exámenes o los test se han pasado, los contenidos son borrados de la cabeza de los estudiantes. La cultura es la de "materia pasada, materia olvidada".

Además de eso, en el caso de la Física, por ejemplo, su imagen es totalmente distorsionada. Física es una herencia humana, un derecho de todo(a) ciudadano(a). Sin embargo, la imagen que trae la enseñanza para los test es que Física es privilegio de pocos porque es difícil, formulística, pesada, es decir, solamente para "inteligentes".

A nivel universitario muchos profesores "dan la materia", lo que significa que repiten lo que está en los libros y los estudiantes tienen que resolver las "clásicas listas de problemas”, que muchas veces ya están resueltos en algún sitio.

¿Qué enseñanza es esa? ¿Ineficaz? ¡No! Es anticientífica, como dijo Carl Wieman hace pocos años.

\section{Competencias y conocimientos}

Hoy es muy común hablar de currículum por competencias o enseñanza por competencias. Los primeros significados del concepto de competencia estaban asociados al mundo laboral y tenían que ver con habilidades prácticas.

Con el advenimiento del conductismo, en las escuelas las competencias pasaron a ser los objetivos conductistas que definían claramente lo que los estudiantes deberían ser capaces de hacer al final de una unidad didáctica.

Los significados no eran considerados, lo que se esperaba era que los alumnos presentasen las conductas definidas en los objetivos.

El resultado fue un aprendizaje esencialmente mecánico de contenidos declarativos y procedimentales.

Hoy en día se argumenta que competencia tiene otros significados; sin embargo, todavía reflejan un enfoque conductista en la educación.

Perrenoud (2000), un conocido autor en el campo de las competencias, argumenta que es un error pensar que el desarrollo de competencias dispensa la adquisición de conocimientos.

Competencias evidenciadas en determinada acción no son, por sí solas, conocimiento. El desarrollo significativo de competencias no es independiente de la adquisición significativa de conocimientos.

El currículum por competencias no descarta conocimientos y disciplinas. Sin embargo, la educación, particularmente la enseñanza, independiente del foco en competencias, sigue centrada en el paradigma de la transmisión de conocimientos y su aprendizaje mecánico.

La adquisición mecánica de contenidos es el paradigma, mientras debería ser el aprendizaje significativo de contenidos, dado que el objetivo debería estar volcado 
hacia la adquisición de conocimientos y competencias para la ciudadanía, para la vida.

Hay una relación dialéctica entre la adquisición de conocimientos significativos y competencias. Cuantos más conocimientos se adquiere de modo significativo más competencias son desarrolladas y viceversa.

Eso nos lleva a preguntar qué competencias estarían asociadas a la enseñanza de una verdadera ciencia, dado que la ciencia no está restringida a fórmulas, reacciones, taxonomías y respuestas correctas.

Deberían ser competencias científicas como, por ejemplo, modelado científico, argumentación basada en evidencias, comunicación de resultados. Dichas competencias podrían ser desarrolladas, por ejemplo, a través de laboratorios virtuales que pueden motivar a los estudiantes a cambiar características de los modelos y ver qué pasa y conducir experimentos sobre fenómenos no observables directamente. Podrían también ser desarrolladas simulaciones y construcciones de modelos computacionales.

El desarrollo de competencias no puede estar restringido a llenar un cerebro de conocimientos, pero sí a desarrollar ese cerebro (Wieman, 2013).

\section{¿Qué es aprendizaje Significativo?}

Es adquisición de conocimientos con significado, con comprensión. Es saber decir y saber hacer; es ser capaz de explicar, de describir, de aplicar conocimientos, incluso a situaciones nuevas, pero siempre con significado.

La interacción cognitiva entre conocimientos nuevos y previos es la característica clave del aprendizaje significativo.

En dicha interacción el nuevo conocimiento debe relacionarse de manera no arbitraria y no literal con aquello que el aprendiz ya sabe.

E1 papel del conocimiento previo

Si tuviese que reducir toda la psicología educativa a un solo principio, enunciaría este (Ausubel, 1978):

De todos los factores que influyen en el aprendizaje, el más importante es lo que el alumno ya sabe. Averígüese eso y enséñese de acuerdo con ello.

\section{La intencionalidad}

Además del importante rol del conocimiento previo, otra condición para la ocurrencia del aprendizaje significativo es que el aprendiz presente una predisposición para aprender, una intencionalidad, un querer aprender, un interés.

Desafortunadamente, la enseñanza de las ciencias, en general, no despierta esa intencionalidad en los alumnos.

\section{Aprendizaje Mecánico}

Es el aprendizaje netamente memorístico, sin significado, sin comprensión, sin capacidad de explicar.

Sirve para reproducir, a corto plazo, respuestas en pruebas cuando la materia es la misma que "fue dada" por el profesor en las clases.

Es el que predomina en la enseñanza de las ciencias. 


\section{AS y la enseñanza de las Ciencias}

¿La enseñanza de las ciencias tiene en cuenta esas dos condiciones básicas?

Desafortunadamente, ¡no! Lo que predomina en la educación básica y superior es el aprendizaje mecánico, sin significado, de fórmulas y definiciones, leyes y teorías que son aplicadas a situaciones conocidas, sin comprensión.

Eso es muy triste porque el aprendizaje de ciencias es una herencia científica que todos los seres humanos tienen el derecho de adquirir significativamente.

Es reconocido internacionalmente que la mayoría de los estudiantes desarrollan, o vienen con él a la escuela, un sentimiento negativo respecto a las ciencias, difíciles, aburridas, hay que memorizar muchos contenidos mecánicamente.

La perspectiva psicológica humanista tiene como principio básico que el ser humano integra pensamientos, sentimientos y acciones para bien o para mal.

¿Por qué entonces la integración es tan frecuentemente negativa respecto a las ciencias? ¿Por qué las ciencias no son percibidas como relevantes para la ciudadanía? ¿Cómo cambiar ese escenario?

Hay muchas respuestas para esas preguntas. Por ejemplo, se puede cambiar el currículum de modo que incorpore Ciencia Moderna y Contemporánea, así como las Tecnologías Digitales de Información y Comunicación, aprendizaje activo, enseñanza centrada en el alumno, actividades colaborativas, secuencias de enseñanza y aprendizaje, games.

Sin embargo, independiente del significado de cada una de esas posibles respuestas, cuando el objetivo es facilitar el aprendizaje significativo de contenidos científicos, de alguna manera se debe considerar el conocimiento previo del estudiante y despertar su intencionalidad para aprender ciencias.

No tiene ningún sentido empezar a enseñar un contenido dado de un cuerpo de conocimientos sin ninguna idea de lo que ya saben, o no saben, los alumnos respecto a ese contenido.

Otra cosa que no tiene sentido es empezar a enseñar un contenido sin utilizar situaciones que tengan sentido para el alumno, que sean de su mundo, de su cultura.

\section{Situaciones y conceptos}

Son las situaciones las que dan sentido a los conceptos y la conceptualización está en el núcleo del desarrollo cognitivo (Vergnaud, 1990).

Conceptos están en la base de la comprensión humana (Toulmin, 1977). Conceptos son estructurantes de campos de conocimiento. Sin el concepto de campo el Electromagnetismo no existiría. Sin el concepto de rayo de luz la Óptica Geométrica no existiría. Y por ahí va.

Las primeras situaciones deben ser del contexto del alumno, deben tener sentido para el alumno y despertar su predisposición, su intencionalidad para aprender.

Es un error empezar la enseñanza sin usar situaciones que tengan sentido para los alumnos. Desafortunadamente es un error muy común en la enseñanza de las ciencias. ¿Cómo esperar que a los estudiantes les gusten las ciencias?

Lo mismo pasa en la enseñanza superior. Por ejemplo, en disciplinas de Física General y de Cálculo para futuros ingenieros no se utilizan situaciones de la Ingeniería. 
El resultado es que a los estudiantes de Ingeniería lo único que les importa es pasar y verse libre de la Física y del Cálculo. Un absurdo

En la enseñanza de las ciencias, conceptos y situaciones reciben mucho menos atención que las fórmulas. Sin embargo, no tiene sentido usar fórmulas, ecuaciones, tablas, reacciones, clasificaciones, organigramas mecánicamente sin comprender los conceptos involucrados.

Pensando en la relevancia del conocimiento científico para la ciudadanía es fácil concluir que la enseñanza de las ciencias debería ser diferente, estimulando conceptualización, modelado, experimentación, argumentación basada en evidencias.

\section{Modelos en la Física, como ejemplo}

Modelos, así como conceptos están en el corazón de la Física. El Universo es complejo, pero la Física desarrolla modelos con aproximaciones que nos permiten lidiar con esa complejidad.

La Física no es una ciencia exacta dado que sus teorías están basadas en modelos que incluyen aproximaciones. Sin embargo, eso no significa disminuir la Física. Al revés, los modelos de la Física son muy buenos y ayudan a entender fenómenos físicos desde el micro al macrocosmo.

\section{Modelos en la enseñanza}

No obstante, en la enseñanza de las ciencias modelos y su construcción son ignorados. Las teorías son enseñadas como descubrimientos de genios y como si fueran definitivas. Eso no es ciencia y mucho menos para la ciudadanía.

Modelos y modelaje deben integrar naturalmente la enseñanza de las ciencias. Es mucho más importante aprender a modelar científicamente que aprender fórmulas, ecuaciones, taxonomías y aplicarlas mecánicamente a situaciones conocidas.

En la actualidad el modelado computacional es una gran posibilidad para "otra enseñanza de las ciencias", pero la enseñanza para los test (el teaching for testing) no permite eso porque sería una "pérdida de tiempo".

\section{Criticidad y dialogicidad}

En la enseñanza tradicional, tipo "bancaria" (los conocimientos son "depositados" en la cabeza del alumno), lo que se espera de los estudiantes es que reproduzcan literalmente los conocimientos que fueron depositados en sus cabezas. Significados y comprensión no son requisitos.

Este tipo de enseñanza inhibe la criticidad y el cuestionamiento. Sin embargo, en las ciencias, criticidad y dialogicidad son intrínsecas a esas disciplinas. Preguntas y pensamiento crítico están siempre presentes en las ciencias.

Estudiantes que no aprenden a preguntar, a cuestionar, no están aprendiendo ciencias. Sin embargo, la educación bancaria, la enseñanza para los test, no estimula eso.

Aprendizaje de las ciencias como un derecho del ciudadano no está asociado a esta pasividad de la educación bancaria, sino a una educación dialógica. 
Dialogicidad está en la esencia de una educación libertadora. Implica que el aprendizaje desarrolle una conciencia crítica. Que no acepte soluciones mágicas, que verifique explicaciones y que esté siempre abierta a revisiones (Freire, 1988). Las ciencias son así, pero su enseñanza ignora esas características básicas y las transforma en una obligación netamente memorística.

\section{Aprendizaje significativo crítico}

Es aprendizaje con significado y con criticidad, con conciencia crítica. Es captar los significados de los conocimientos que están siendo trabajados, pero no como únicos o definitivos. Lo mismo con teorías, modelos, respuestas. Ningún conocimiento es único y definitivo.

Para un aprendizaje significativo crítico a través de la enseñanza de las ciencias se recomienda (Moreira, 2005). Algunos de sus principios son:

- Enseñar /aprender a preguntar en vez de memorizar respuestas "correctas". (Principio de la interacción social y del cuestionamiento).

- Enseñar/ aprender a través de distintos materiales educativos, no solamente el libro de texto. (Principio de la no centralidad del libro de texto).

- Aprender que somos perceptores y representadores del mundo. (Principio del aprendiz como perceptor y representador).

- Aprender que el lenguaje está totalmente involucrado en cualquier intento de percibir la realidad. (Principio del conocimiento como lenguaje).

- Aprender que el significado está en las personas no en las palabras, en las cosas. (Principio de la conciencia semántica).

- Aprender que seres humanos aprenden corrigiendo sus errores. (Principio del aprendizaje por el error).

- Aprender a desaprender, a no usar conceptos y estrategias irrelevantes. (Principio del desaprendizaje).

- Aprender que preguntas son instrumentos de percepción y que definiciones y metáforas son instrumentos para pensar. (Principio de la incertidumbre del conocimiento).

- Aprender a partir de distintas estrategias de enseñanza. (Principio del abandono de la narrativa y de la pizarra).

\section{Algunas tendencias contemporáneas}

En continuidad se presentan algunas propuestas actuales para la enseñanza de las ciencias. Son tendencias contemporáneas. Sin embargo, todavía no hay evidencias convincentes de que mejoran la enseñanza y el aprendizaje de las ciencias. Hay mucho que investigar en ese campo.

\section{What's personalized learning? (Harold, 2016)}

Many in the ed-tech field see new technologies as powerful tool to help schools meet the needs of ever-more-diverse student populations. The idea is that digital devices, software, and learning plat- 
forms offer a once-unimaginable array of options for tailoring education to each individual student's academic strengths and weaknesses, interests and motivations, personal preference, and optimal pace of learning.

In recent years, a group of organizations including the Bill \& Melinda Gates Foundation, the Michael and Susan Dell Foundation, and EDUCAUSE have crafted a definition of "personalized learning" that rests on four pillars.

- each student should have a "learn profile" that documents his or her strengths, weaknesses, preferences, and goals;

- each student should pursue an individualizad learning path that encourages him or her to set and manage personal academic goals;

- students should follow a "competency-based progression" that focuses on their ability to demonstrate mastery of a topic, rather than seat time; and,

- students learning environments should be flexible and structured in ways that support their individual goals.

Despite the massive investments that many school systems are making, the evidence that digital personalized learning can improve student outcomes or narrow achievement gaps at scale remains scattered, at best.

\section{Blended learning (ibid.)}

The basic premise involves students rotating between online and in-person stations for different parts of the day. There are many versions of this approach, however: Do students stay in the classroom or go to a computer lab?

Does on line instruction cover core content, or is it primarily for remediation? Are all students doing the same thing on line, or different students have different software and learning experiences?

As of now, there is no definitive evidence that blended learning works (or doesn't). While some studies have found encouraging results with specific programs or under certain circumstances, the question for whether blended learning positively impacts student learning still has a mostly unsatisfactory answer: "It depends."

\section{Deep learning (ibid.)}

At the same time that students are facing a more challenging world, the days of set knowledge and accomplishment based on content are over.

Deep learning is the process of acquiring six global competencies: character, citizenship, collaboration, communication, creativity, and critical thinking

\section{Conclusión}

Desafortunadamente, vivimos en una cultura en la que la educación, básica y superior, está volcada hacia el mercado, no a la ciudadanía.

Las escuelas son más centros de entrenamiento que centros de educación. 
La enseñanza de las ciencias está inserta en esa cultura, basada en la memorización de fórmulas, reacciones, taxonomías ... y respuestas correctas.

Las ciencias pueden contribuir mucho a la ciudadanía, para la vida de los (as) ciudadanos(as), porque están en la base de la tecnología, de la medicina, ...

Además, las ciencias pueden llevar a los ciudadanos(as) excelentes modelos y teorías que son de su mundo.

Ciencia es pensar, cuestionar, argumentar, experimentar, teorizar, comunicar resultados

Sin embargo, la enseñanza de las ciencias ignora todo eso y no contribuye a una formación para la ciudadanía. Focaliza mucho más en la preparación para los test y para el mercado.

Esa enseñanza debe ser repensada. Ciencia es mucho más que eso. Así como lo es una enseñanza para la ciudadanía.

Enseñanza para los test (el tracking foro testigo) es un insulto a la ciencia y a la ciudadanía.

\section{Referencias}

Ausubel, D. P. (1978). Psicología educativa: Un punto de vista cognoscitivo. México: Editorial Trillas. [Trad. al español del original en inglés Educational psychology: A cognitiveview (1968)]. Holt, Rinehart, and Winston.

Freire, P. (1988). Pedagogia do oprimido. (18 ${ }^{\underline{a}}$ edição). Paz e Terra.

Harold, B. (2000). Technology in Education: An overview. Education Week. February 5. Moreira, M. A. (2005). Aprendizagem Significativo Crítico. Instituto de Física da UFRGS. Perrenoud, P. (2000). Novas competências para ensinar. Artmed.

Science (2013). Grand Challenges in Science Education, 340, 290-323.

Toulmin, S. (1977). La comprensión bumana, Vol. 1: El uso colectivo y la evolución de los conceptos. Alianza.

Vergnaud, G. (1990). La théorie des champs conceptuels. Récherches en Didatique des Mathématiques, 10(23), 133-170. 